\title{
reg $T$ can modulate gingipain activity and response to oxidative stress in Porphyromonas gingivalis
}

\begin{abstract}
Correspondence
H. M. Fletcher

hfletcher@llu.edu
\end{abstract}

Received 18 January 2010

Revised 23 June 2010

Accepted 24 June 2010

\author{
E. Vanterpool, ${ }^{1}$ A. Wilson Aruni, ${ }^{2}$ F. Roy ${ }^{2}$ and H. M. Fletcher $^{2}$ \\ ${ }^{1}$ Department of Biological Sciences, Oakwood University, Huntsville, AL 35896, USA \\ ${ }^{2}$ Department of Basic Sciences, Division of Microbiology and Molecular Genetics, \\ School of Medicine, Loma Linda University, Loma Linda, CA 92350, USA
}

\section{INTRODUCTION}

The capacity of micro-organisms to rapidly and specifically adapt to environmental conditions is an essential characteristic for their pathogenic potential (Bronner et al., 2004; Dorman, 2009). While adaptation is mostly regulated by complex networks that may act at the transcriptional level, post-translational control of several virulence factors is important in the host-microbe interaction. Porphyromonas gingivalis, a Gram-negative, anaerobic bacterium, has been shown to be associated with periodontal disease and other systemic diseases, including cardiovascular disease (Lamont \& Jenkinson, 1998; Seymour et al., 2007; Amano, 2003). The significance and environmental regulation of several of the major virulence factors of $P$. gingivalis, including the gingipains, are well documented (Abaibou et al., 2001; Hasegawa et al., 2003; Mikolajczyk et al., 2003; Potempa et al., 2003; Vanterpool et al., 2004; Fitzpatrick et al., 2009; Curtis et al., 2005). The gingipains are extracellular and/or cell-associated. The Arg-specific gingipains, RgpA and $\mathrm{RgpB}$, are encoded by the genes $\operatorname{rgp} A$ and $\operatorname{rgp} B$ respectively, whereas the Lys-specific protease (Kgp) is encoded by one gene, $k g p$ (Nakayama, 2003). There is still a gap in our understanding of post-translational control of these factors

Four supplementary figures are available with the online version of this paper. and their potential impact on the pathogenicity of this organism.

In $P$. gingivalis there is emerging evidence of a unique regulatory mechanism(s) for gingipain biogenesis. Several genes, including vimA, vimE, vimF, gppX, porR, sovPG27 and $\operatorname{por} T$, have been shown to play regulatory roles in gingipain activation and/or maturation (Abaibou et al., 2001; Hasegawa et al., 2003; Ishiguro et al., 2009; Saiki \& Konishi, 2010; Vanterpool et al., 2004, 2005, 2006). In $P$. gingivalis FLL92, a vimA-defective mutant, we previously reported a late onset of proteolytic activity during the late exponential phase and altered gingipain distribution even during the stationary phase. In addition, a $64 \mathrm{kDa}$ partially processed $\operatorname{RgpB}$ that was altered in its glycosylation was secreted from the vimA-defective mutant (Olango et al., 2003; Vanterpool et al., 2006). Protein-protein interaction studies using the purified $\mathrm{rVimA}$ showed that this protein interacts with the gingipains, HtrA, PG2096 [Oralgen database gene ID: PG1833 (http://www.oralgen.lanl.gov/)] and other proteins in P. gingivalis (Roy et al., 2006; Vanterpool et al. 2004). PG2096 appears to have homology with other $G$ proteins; however, its role in gingipain regulation and virulence in $P$. gingivalis is unclear.

The involvement of $G$ proteins as regulatory molecular switches in the signal transduction pathways that may modulate virulence and environmental stress has been 
demonstrated in several bacteria (Baev et al., 1999; Mauriello et al., 2010; Scott \& Haldenwang, 1999). The annotated genomic database for $P$. gingivalis has identified several putative genes (PG1241, PG2142, PG2143, PG0346, PG0048 and PG0711) that may have G-protein-like properties and could modulate various stress functions such as protein intake and transport of iron and iron-copper cluster regulation (http://www.oralgen.lanl.gov/). Here we report that PG2096, designated regT (regulator of gingipain activity at elevated temperatures), plays a role in regulating the gingipains at elevated temperatures. RegT is also involved in resistance to oxidative stress in $P$. gingivalis.

\section{METHODS}

In silico analysis. Nucleotide sequences for Porphyromonas gingivalis and all the other strains described below were obtained from the Oralgen database (http://www.oralgen.lanl.gov/) and NCBI web server (http://www.ncbi.nlm.nih.gov/), respectively. The sequences were analysed using CLUSTAL W (Larkin et al., 2007) and Lasergene Version 8 (Burland, 2000).

Bacterial strains and growth conditions. Strains and plasmids used in this study are listed in Table 1. $P$. gingivalis strains were grown in Brain Heart Infusion (BHI) broth (Difco) supplemented with haemin $\left(5 \mu \mathrm{g} \mathrm{ml}^{-1}\right)$, vitamin $\mathrm{K}\left(0.5 \mu \mathrm{g} \mathrm{ml}^{-1}\right)$ and cysteine $(0.1 \%)$. Experiments with hydrogen peroxide were performed in BHI without cysteine. Escherichia coli strains were grown in Luria-Bertani broth. Unless otherwise stated, all cultures were incubated at $37^{\circ} \mathrm{C} . P$. gingivalis strains were maintained in an anaerobic chamber (Coy Manufacturing) in $10 \% \mathrm{H}_{2} / 10 \% \mathrm{CO}_{2} / 80 \% \mathrm{~N}_{2}$. Growth rates for $P$. gingivalis and E. coli strains were determined spectrophotometrically $\left(\mathrm{OD}_{600}\right)$. Antibiotics were used at the following concentrations: clindamycin, $0.5 \mu \mathrm{g} \mathrm{ml}^{-1}$; erythromycin, $300 \mu \mathrm{g} \mathrm{ml}^{-1}$; carbenicillin, $100 \mu \mathrm{g} \mathrm{ml}^{-1}$.

DNA isolation and analysis. $P$. gingivalis chromosomal DNA was prepared as described by Marmur (1961). For plasmid DNA analysis, DNA extraction was performed by the alkaline lysis procedure as previously reported (Vanterpool et al., 2004). For large-scale preparation, plasmids were purified using the Qiagen plasmid maxi kit.

Generation of a PG2096-defective mutant $\boldsymbol{P}$. gingivalis strain. A $4.7 \mathrm{~kb}$ fragment carrying the intact PG2096 ( $\mathrm{reg} T$ ) open reading frame was amplified by PCR using the oligonucleotide primers P1 and
P2 (Table 2). This fragment was cloned into the pCR2.1-TOPO plasmid vector (Invitrogen) and designated pFLL99. The recombinant plasmid was digested with HincII, to remove an internal $3.1 \mathrm{~kb}$ fragment of the gene. The ermF-ermAM cassette which confers erythromycin/clindamycin resistance in E. coli and $P$. gingivalis was PCR amplified from pVA2198 using Pfu turbo (Stratagene) and inserted into the HincII site using standard methods (Sambrook \& Russell, 2001). Orientation of the erythromycin cassette was determined by restriction endonuclease analysis. The resultant recombinant plasmid, pFLL204, was used as a donor in electroporation of $P$. gingivalis W83 as previously reported (Fletcher et al., 1995; Vanterpool et al., 2004). Confirmation of the regT-defective mutant was carried out by performing PCR using regT-specific primers, which amplified a $3.7 \mathrm{~kb}$ amplicon from the mutant in contrast to a $4.7 \mathrm{~kb}$ amplicon from the wild-type strain. erm-specific primers (Table 2) were used to confirm the amplification of a $2.1 \mathrm{~kb}$ amplicon specific for the erythromycin cassette.

Growth analysis at elevated temperatures and under oxidative stress conditions. Actively growing cultures of $P$. gingivalis wildtype W83 and the PG2096-defective mutant FLL205 were incubated at $42{ }^{\circ} \mathrm{C}$ under anaerobic conditions for $28 \mathrm{~h}$. Growth was determined by $\mathrm{OD}_{600}$ readings at $0,4,8,24$ and $28 \mathrm{~h}$. For adaptation to oxidative stress conditions, the strains were grown in $\mathrm{BHI}$ without cysteine in the presence of $0.25 \mathrm{mM} \mathrm{H}_{2} \mathrm{O}_{2}$. Controls were grown in the absence of $\mathrm{H}_{2} \mathrm{O}_{2}$. Growth was determined by $\mathrm{OD}_{600}$ readings at $0,2,4,6,24$ and $28 \mathrm{~h}$.

Protease activity under environmental stress conditions. Protease activity was determined as previously reported (Sheets et al., 2006) for cells grown to exponential phase $\left(\mathrm{OD}_{600} 0.8\right)$ and stationary phase $\left(\mathrm{OD}_{600} 1.2\right)$. For analysis of gingipain activity of $P$. gingivalis strains at elevated temperatures, cells were incubated at $42{ }^{\circ} \mathrm{C}$ under anaerobic conditions for $28 \mathrm{~h}$.

SDS-PAGE and immunoblot analysis. SDS-PAGE was performed with a $4-12 \%$ Bistris separating gel in MOPS-SDS running buffer according to the manufacturer's instructions (NuPAGE Novex gels; Invitrogen). Samples were prepared (65\% sample, $25 \% 4 \times$ NuPAGE LDS sample buffer, $10 \%$ NuPAGE reducing agent), heated at $72{ }^{\circ} \mathrm{C}$ for $10 \mathrm{~min}$, and then electrophoresed at $200 \mathrm{~V}$ for $65 \mathrm{~min}$ in the XCell SureLock Mini-Cell System (Invitrogen). The protein bands were visualized by staining with Simply Blue Safe stain (Invitrogen). The separated proteins were then transferred to BioTrace nitrocellulose membranes (Pall Corporation) and processed at $15 \mathrm{~V}$ for $25 \mathrm{~min}$ with a Semi-Dry Trans-Blot apparatus (Bio-Rad). The blots were probed with gingipain-specific antibodies (Potempa et al., 1998).

Table 1. Plasmids and bacterial strains

\begin{tabular}{|c|c|c|}
\hline Strain or plasmid & Phenotype/description & Source \\
\hline \multicolumn{3}{|l|}{ Plasmids } \\
\hline pCR2.1-TOPO & $\mathrm{Ap}^{\mathrm{r}} \mathrm{Km}^{\mathrm{r}}$ & Invitrogen \\
\hline pFLL99 & pCR2.1-TOPO : PG2096 & This study \\
\hline pFLL204 & pCR2.1-TOPO : PG2096: : ermF-ermAM & This study \\
\hline pVA2198 & $\mathrm{Sp}^{\mathrm{r}}$, ermF-ermAM & Fletcher et al. (1995) \\
\hline \multicolumn{3}{|l|}{ P. gingivalis } \\
\hline W83 & Wild-type & This study \\
\hline FLL205 & regT-defective mutant & This study \\
\hline \multicolumn{3}{|l|}{ E. coli } \\
\hline $\mathrm{DH} 5 \alpha$ & $\begin{array}{c}\mathrm{F}^{-} \phi 80 \mathrm{~d} l a c Z \Delta \mathrm{M} 15 \Delta(\text { lacZYA-argF }) \text { U169 recA1 endA1 } \\
\text { hsdR17 }\left(\mathrm{r}_{\mathrm{k}}^{-} \mathrm{m}_{\mathrm{k}}^{+}\right) \text {phoA supE44 } \lambda^{-} \text {thi-1 gyrA96 relA1 }\end{array}$ & Invitrogen \\
\hline
\end{tabular}


Table 2. Primers

\begin{tabular}{|ll|}
\hline Primer & \multicolumn{1}{c|}{ Sequence $\left(\mathbf{5}^{\prime}-\mathbf{3}^{\prime}\right)$} \\
\hline P1 PG2096 forward & ATGGGTCGTATCAAGCAACGT \\
P2 PG2096 reverse & TCGCTTTGTCGGAATGATAT \\
P3 $\mathrm{erm}$ forward & TATTAGGCCTATAGCTTCCGCTATT \\
P4 $\mathrm{erm}$ reverse & AATAGGCCTTAGTAACGTGTAACTTT \\
\hline
\end{tabular}

Immunoreactive proteins were detected by the procedure described in the Western Lightning Chemiluminescence Reagent Plus kit (PerkinElmer Life Sciences). The secondary antibody was goat anti-rabbit or anti-chicken IgG alkaline (heavy plus light chains)-horseradish peroxidase conjugate (Zymed Laboratories).

Analysis of sialidase activity. Strains were assessed for their sialidase activities using the Amplex Red Sialidase Activity kit according to the manufacturer's protocol (Invitrogen).

\section{RESULTS}

\section{In silico analysis of PG2096}

PG2096 (regT) shows $62 \%$ homology with the gene for the Sgp G protein from Streptococcus mutans (Fig. 1). These genes also clustered with $P G 0411$, which encodes a possible outermembrane protein in $P$. gingivalis. The six annotated genes that encode $G$ proteins in $P$. gingivalis formed a separate cluster (see Supplementary Fig. S1, available with the online version of this paper). The Sgp G protein from S. mutans shares structural similarity with $G$ proteins from Pseudomonas aeruginosa and E. coli (Baev et al., 2000). CLUSTAL W analysis using amino acid sequences showed similarity between RegT and other G proteins from $P$. gingivalis, $P$. aeruginosa, E. coli and S. mutans (Supplementary Fig. S2). RegT was more closely related to the $P$. aeruginosa lineage. In the PG2096 protein there are eight conserved G-protein-like regions between positions 418-429, 460, 515-518, 588, 629-631, 645, 689-690 and 717 (Fig. 1, Supplementary Fig. S3). There is also conserved domain architecture that is similar to that of other hypothetical proteins found in Bacteroides fragilis (gi 81443196, gi 81313587), Treponema denticola (gi 81411469) and Prevotella spp (gi 281300483) (Geer et al., 2002). It is noteworthy that while PG0411 is highly homologous to RegT, it lacks the conserved G-protein-like domains, but shows domain similarity with the TonB, PorT, Kgp and RgpB proteins (Aravind \& Koonin, 1999). These domains are missing in the RegT protein. No DNA-binding domains were identified in RegT; however, several RNA-binding sites were predicted (data not shown).

\section{Inactivation of the regT gene in $P$. gingivalis W83 by allelic-exchange mutagenesis and confirmation by RT-PCR}

Isogenic mutants of $P$. gingivalis W83 defective in the PG2096 gene (designated regT) were constructed by allelicexchange mutagenesis. The circular recombinant plasmid pFLL204, which carries the ermF-ermAM cassette in the HincII restriction site ( $0.8 \mathrm{~kb}$ of the open reading frame) of the regT gene, was used as a donor in electroporation of $P$. gingivalis W83. Following electroporation and plating on selective medium (BHI containing $10 \mu \mathrm{g}$ erythromycin $\mathrm{ml}^{-1}$ ), we detected approximately 70 erythromycinresistant colonies after a 6 day incubation period. To compare their phenotypic properties with those of wildtype strain W83, all mutants were plated on Brucella blood agar plates. Similar to the wild-type strain, all mutants had a black-pigmented and $\beta$-haemolytic phenotype. Chromosomal DNA from two randomly chosen
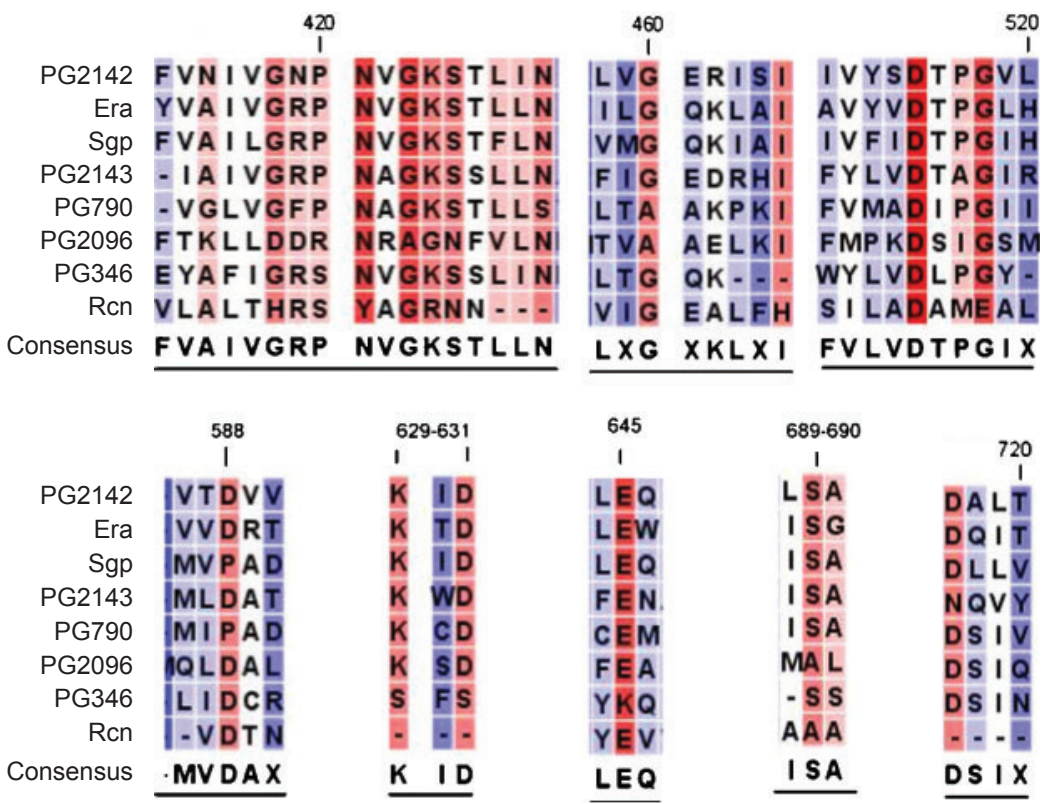

\begin{tabular}{|c|c|}
\hline 689.690 & \\
\hline 1 & 720 \\
\hline LSA & DALT \\
\hline ISG & DQIT \\
\hline ISA & DL L \\
\hline ISA & $\mathrm{NQV} Y$ \\
\hline ISA & DS IV \\
\hline MA L & DS I \\
\hline$-s s$ & DS I N \\
\hline AAA &.-- \\
\hline ISA & DS I $x$ \\
\hline
\end{tabular}

Fig. 1. Multiple sequence alignment of the amino acid sequences showing conserved Gprotein-like domains in relation to the $G$ protein sequences from $P$. gingivalis and other bacteria. 
erythromycin-resistant colonies and the wild-type were analysed using PCR to confirm the inactivation in the regT gene. If the regT gene was interrupted by the ermF-ermAM cassette, a $3.7 \mathrm{~kb}$ fragment was expected to be amplified using primers $\mathrm{P} 1$ and $\mathrm{P} 2$ (Table 2). The expected $3.7 \mathrm{~kb}$ and $4.7 \mathrm{~kb}$ fragments were observed in the two erythromycin-resistant strains and the wild-type W83, respectively. Using erm primers should amplify the $2.1 \mathrm{~kb}$ ermFermAM cassette from the regT-defective mutants. PCR analysis using erm cassette primers yielded an amplified $2.1 \mathrm{~kb}$ fragment as predicted. RT-PCR analysis of the defective mutant showed the absence of the regT fragment in the regT-isogenic defective mutant in contrast to its presence in the wild-type (data not shown). Taken together, these results indicated the insertional inactivation of the regT gene with the $2.1 \mathrm{~kb}$ ermF-ermAM antibiotic cassette. One mutant, designated $P$. gingivalis FLL205, was randomly chosen for further study.

\section{Growth analysis under thermal and oxidative stress}

To determine if regT played a role in growth of $P$. gingivalis at elevated temperatures, strains W83 and FLL205 were incubated at $42{ }^{\circ} \mathrm{C}$ under anaerobic conditions for $28 \mathrm{~h}$. There was no significant difference $(P \geqslant 0.1)$ in the growth in the regT-defective mutant and the wild-type when grown at $42{ }^{\circ} \mathrm{C}$ up to $24 \mathrm{~h}$ (Fig. 2). However, autolysis of FLL205 was somewhat increased when compared to the parent strain at $28 \mathrm{~h}$ (Fig. 2). To determine if regT played a role in regulating $P$. gingivalis growth under oxidative stress, the regT-defective mutant and wild-type were grown in the presence of $0.25 \mathrm{mM} \mathrm{H}_{2} \mathrm{O}_{2}$. As shown in Fig. 3, the regT-defective mutant appeared to be more resistant to $0.25 \mathrm{mM} \mathrm{H}_{2} \mathrm{O}_{2}$ in comparison to the wild-type, although

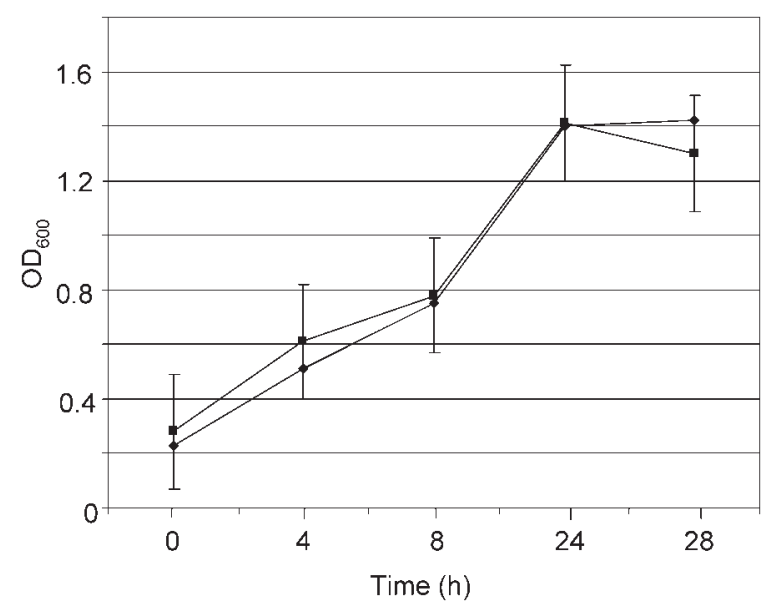

Fig. 2. Growth of $P$. gingivalis FLL205 is not affected at elevated temperature. Cultures of strains W83 (wild-type; $>$ ) and FLL205 (regT-defective; $\square$ ) were grown at $42{ }^{\circ} \mathrm{C}$ for $28 \mathrm{~h}$ under anaerobic conditions. Means \pm SEM $(n=3)$ are plotted. (a)

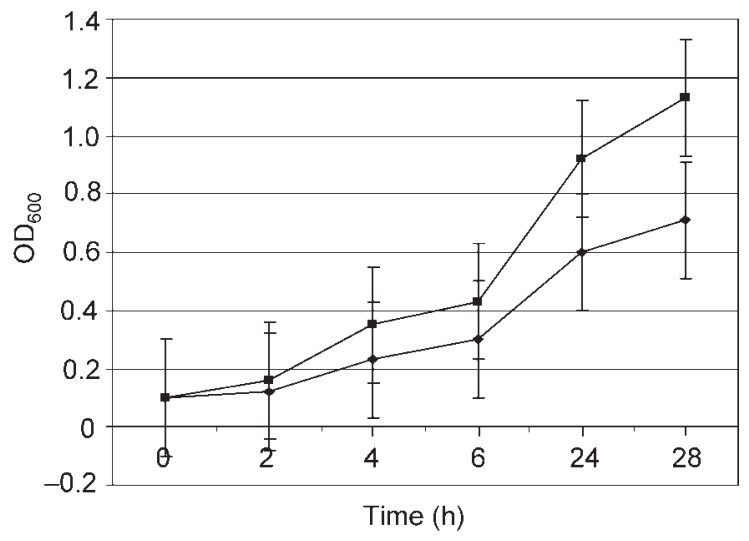

(b)

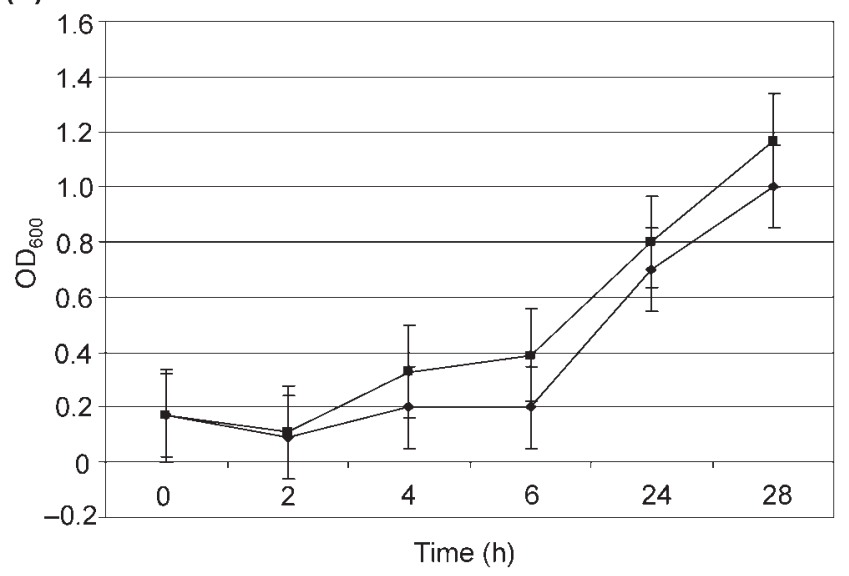

Fig. 3. The regT-defective mutant is more resistant to hydrogen peroxide than the wild-type. Actively growing $P$. gingivalis W83 (a) and FLL205 (b) were incubated with $0.25 \mathrm{mM}$ hydrogen peroxide in the absence of cysteine and growth was measured over $28 \mathrm{~h}$. $\mathbf{\square}$, Hydrogen peroxide-treated; $\boldsymbol{\gamma}$, untreated controls. Means \pm SEM $(n=3)$ are plotted.

the differences between treated and untreated cells were not significant $(P \geqslant 0.1)$ at most time points.

\section{Protease activity under normal and elevated temperature conditions}

To determine if $\operatorname{reg} T$ can affect proteolytic activity, $P$. gingivalis FLL205 and the wild-type were evaluated for gingipain activity at different growth temperatures. There was a $35 \%$ and $21 \%$ decrease in Rgp and Kgp activities, respectively, in $P$. gingivalis FLL205 compared to the wildtype strain grown to stationary phase at $37^{\circ} \mathrm{C}$. A timecourse of gingipain activities at $42{ }^{\circ} \mathrm{C}$ was further evaluated. In FLL205 after $4 \mathrm{~h}$ of incubation, there was a $25 \%$ and $17 \%$ increase in Rgp and Kgp activities, respectively in comparison to the wild-type grown under similar conditions (Fig. 4). After $8 \mathrm{~h}$ of incubation, there was a $48 \%$ increase in Rgp activity (Fig. 4a) and $45 \%$ increase in Kgp activity (Fig. 4b) in FLL205 in comparison 


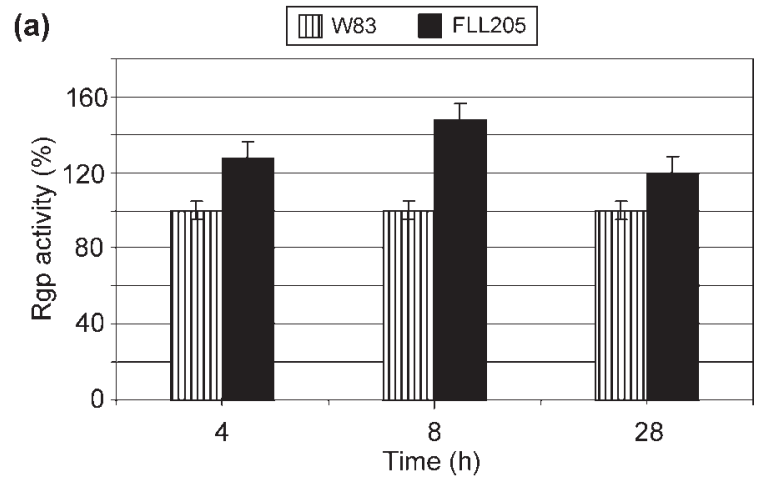

(b)

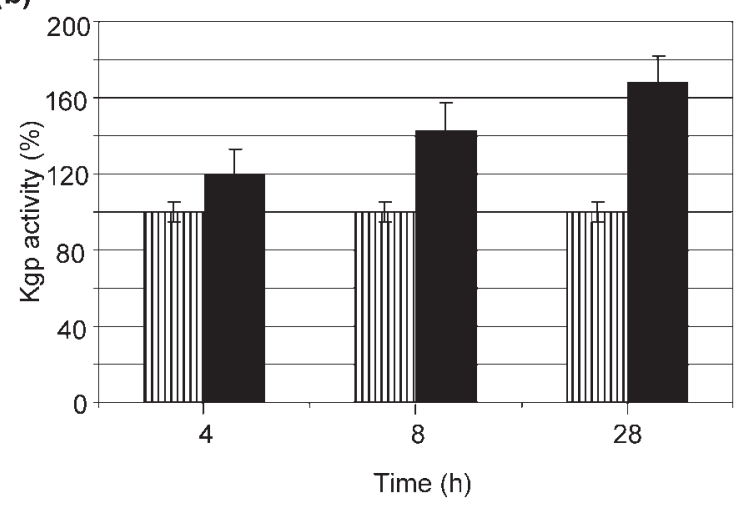

Fig. 4. Gingipain regulation under prolonged exposure to elevated temperatures. $P$. gingivalis W83 and FLL205 were grown at $42{ }^{\circ} \mathrm{C}$ for $28 \mathrm{~h}$. Rgp and Kgp activities were assessed at 4, 8 and $28 \mathrm{~h}$ by hydrolysis of BApNA (a) or ALNA (b), respectively. Means $\pm \operatorname{SEM}(n=3)$ are plotted.

to the wild-type. After $28 \mathrm{~h}$, the Rgp activity in FLL205 returned to a level similar to that seen at the $4 \mathrm{~h}$ time point (Fig. 4a). The Kgp activity, however, increased by $69 \%$ in FLL205 in comparison to the wild-type (Fig. 4b).

Immunoblot analysis using specific antibodies to the extracellular gingipains showed that at $37^{\circ} \mathrm{C}$ the haemagglutinin domains for Rgp and Kgp were missing in FLL205 (Fig. 5a, arrow). Immunoblot analysis of the secreted RgpA and $\mathrm{RgpB}$ from cells grown at $42{ }^{\circ} \mathrm{C}$ showed that the catalytic domain (a $48 \mathrm{kDa}$ immunoreactive band) was more stable in the regT-defective isogenic mutant compared to the wild-type strain (Fig. 5b, arrowed). In addition, the RgpB and Kgp membrane protein profiles of $P$. gingivalis W83 and regT-defective mutant (FLL205) were altered (Fig. 5c). There was no change in the expression of the gingipain genes in the wild-type compared to the regT-defective mutant (Supplementary Fig. S4).

\section{Sialidase activities of the regT-defective mutant}

Because VimA can interact with several other proteins, including a putative sialidase, it is likely that a common protein complex may have pleiotropic regulatory functions (Vanterpool et al., 2006). P. gingivalis W83 and FLL205 were assessed for their sialidase activities. Fetuin was used as the substrate for the sialidase activity assessment. Fetuin contains both the $\alpha 2,3$ and $\alpha 2,6$ linkages of sialic acid residues. A Pg0234 (sialidase)-defective mutant was also generated and used as a comparison for sialidase activity (unpublished data). The sialidase activities from the membrane fractions were not significantly different from that of the wild-type; however, there was a $45 \%$ decrease in secreted sialidase activity in the regT-defective mutant.

\section{DISCUSSION}

Factors that are important in adaptation to environmental stress in the inflammatory microenvironment of the periodontal pocket would be critical for the survival of $P$. gingivalis. In previous reports we showed that the vimA gene can modulate several virulence factors in $P$. gingivalis but not all (Abaibou et al., 2001; Olango et al., 2003; Vanterpool et al., 2006). Furthermore, several other proteins were observed to interact with $\mathrm{rVimA}$ (Abaibou et al., 2001; Vanterpool et al., 2006). These proteins in other systems are known to be involved in posttranslational regulation. HtrA, one such protein, was shown to play a similar role in oxidative and temperature stress in P. gingivalis as observed in other organisms (Roy et al., 2006).

In this study we have further characterized the hypothetical RegT protein, which was also shown to interact with rVimA (Vanterpool et al., 2006). Bioinformatic analysis of RegT has demonstrated G-protein-like properties. This protein was homologous to the other annotated $G$ proteins in $P$. gingivalis and other species including E. coli, $P$. aeruginosa and $S$. mutans (http://www.oralgen.lanl.gov; http://www.ncbi.nlm.nih.gov). Amino acid sequence analysis of RegT revealed eight $\mathrm{G}$ protein conserved domains. Furthermore, there is conserved domain architecture with other hypothetical proteins from other anaerobes, including Bacteriodes fragilis, Treponema denticola and Prevotella spp. The functions of these proteins are still unknown.

$\mathrm{G}$ proteins are known to play an important role in regulating stress-related functions in many organisms (Scott \& Haldenwang, 1999; Jiang et al., 2007). They can act as secondary messengers that change the inactive state of GDP to GTP, which thus triggers downstream cellular processes including the secretion of stress proteins such as heat-shock proteins (Kedzierska et al., 1999). The regulatory role of $\mathrm{G}$ proteins can occur at both the transcriptional and post-transcriptional level (Jiang et al., 2007). At the post-transcriptional level these proteins may modulate mRNA stability, and the assembly of ribosomes and other structural proteins (Sayed et al., 1999). Our study suggests that RegT could be a post-transcriptional regulator. This would be consistent with the absence of any predicted DNA-binding motif. However, the presence of predicted 
(a)

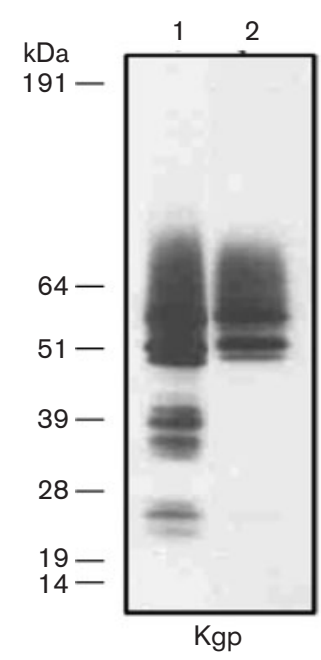

(b)

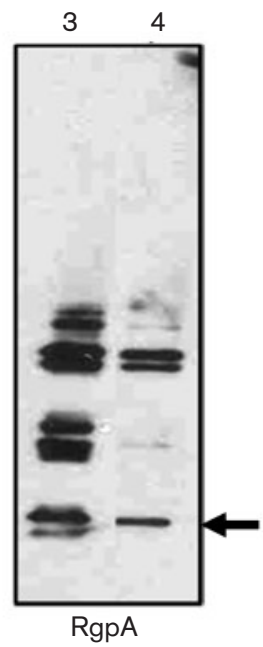

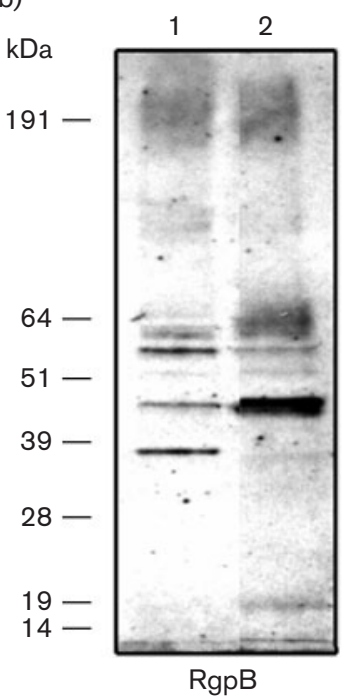

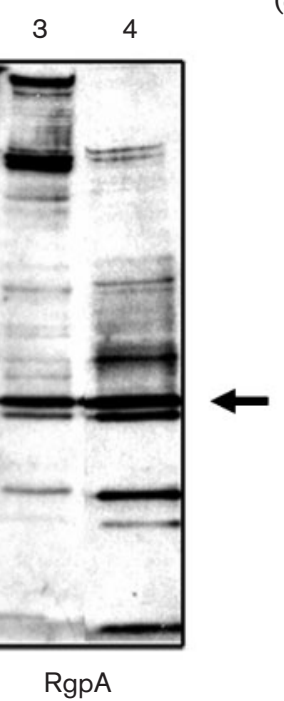

(c)

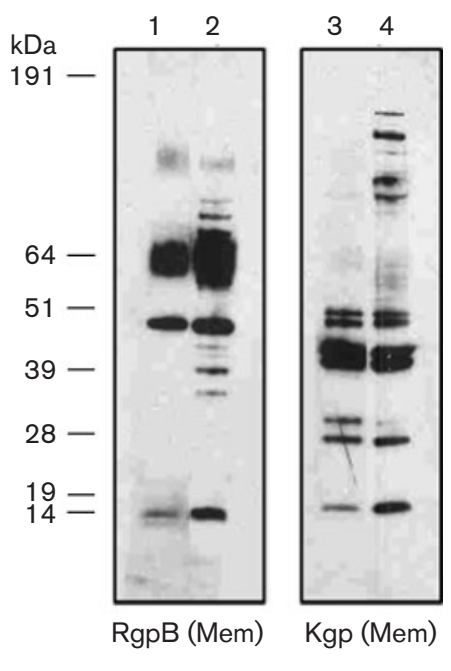

Fig. 5. $(a, b)$ Western immunoblot analysis of the extracellular proteins from $P$. gingivalis using specific anti-RGP and anti-KGP antibodies as probes. All lanes contained $20 \mu \mathrm{g}$ of acetone (60\%)-precipitated proteins from the supernatant fractions of cultures grown in $\mathrm{BHI}$ medium at $37{ }^{\circ} \mathrm{C}$ (a) or $42{ }^{\circ} \mathrm{C} \mathrm{(b)}$. The blots were reacted with antiserum raised in rabbits or chicken against the Arg-X- and Lys-X-specific protease from $P$. gingivalis. Secondary antibody was goat anti-rabbit or anti-chicken lgG alkaline (heavy plus light chains)-horseradish peroxidase conjugate. Lanes 1 and 3, W83; lanes 2 and 4, FLL205. In (a), lanes 1 and 2 were reacted with chicken anti-Kgp; lanes 3 and 4 were reacted with rabbit anti-RgpA. In (b), lanes 1 and 2 were reacted with rabbit anti-RgpB; lanes 3 and 4 were reacted with rabbit anti-RgpA. (c) Immunoblot analysis of membrane fractions. Lanes 1 amd 3, W83; lanes 2 and 4, FLL205. Lanes 1 and 2 were reacted with rabbit anti-RgpB; lanes 3 and 4 were reacted with rabbit anti-Kgp.

RNA-binding sites could raise questions about its role in mRNA stability and ribosome assembly in $P$. gingivalis. This will be further evaluated in the laboratory.

In addition to having elevated temperatures, the periodontal pocket is an oxidative environment due to the presence of reactive oxygen species (Chapple, 1997; Katsuragi et al., 2003; Sculley \& Langley-Evans, 2002). In this study, growth of the regT-defective mutant was not altered at elevated temperatures in comparison to the wildtype. This could suggest that regT does not play a role in survival of the organism under temperature stress. Interestingly, however, the regT-defective mutant was found to be more resistant to oxidative stress compared to the parent strain. These findings are similar to what was observed in the vimA-defective mutant FLL92 (Johnson et al., 2004). Because RegT and VimA can interact, we cannot rule out the possibility that in the vimA-defective mutant, the function of the $\operatorname{reg} T$ gene product could be altered, which would result in the increased resistance to oxidative stress observed in P. gingivalis FLL92 (Johnson et al., 2004). On the other hand, loss of GTPase activity can promote oxidative stress resistance. This would be similar to observations in Saccharomyces cerevisiae, where guanine nucleotide exchange activity promotes resistance to oxidative stress (Olarewaju et al., 2004). Loss of the ability to regenerate active GTP-bound elongation factor favours the cells' ability to respond to oxidative stress. Nucleotide exchange is a critical regulator of most G-proteins. It is unclear if this mechanism is functional in $P$. gingivalis. It is also likely that other genes that may play a role in oxidative stress resistance are upregulated in the regT-defective mutant; this will be the subject of further investigation.

We have also provided evidence that the absence of RegT altered regulation of the gingipains at normal or elevated temperatures. At normal temperature the presence of RegT will facilitate the normal maturation/processing of the gingipains. The increase in gingipain activity at elevated temperatures in FLL205 may result from a more stable catalytic domain which may occur in the absence of RegT. The fact that there was no observable change in the expression of the gingipain genes in the mutant compared to the wild-type suggests that RegT modulation is posttranscriptional. Normally, $P$. gingivalis can modulate its virulence factors to ensure adaptation to its changing host environment (Amano et al., 1994, 2001; Forng et al., 2000; Kesavalu et al., 2003; Murakami et al., 2004; Percival et al., 1999). Because temperature is one of the factors that is known to change as a consequence of an increased inflammatory response in the periodontal pocket (Fedi \& Killoy, 1992), it is likely that RegT is important in regulating gingipain activity in vivo. At increased temperature, a possible downregulation in gingipain activity could influence a decrease in the inflammatory response. Other reports have documented a downregulation of gingipain 
activity at elevated temperatures (Percival et al., 1999). The coordinate downregulation of gingipain activity in response to an environmental cue linked to the intensity of the host inflammatory response would be consistent with the clinically observed cyclical nature of disease progression in periodontal diseases.

In conclusion, we can envision a scenario in $P$. gingivalis where RegT may be important for regulation of gingipain activity and oxidative stress resistance in the inflammatory microenvironment of the periodontal pocket. A specific mechanism for this interaction will be the subject of further study.

\section{ACKNOWLEDGEMENTS}

This work was supported by Loma Linda University and Public Health Grants from NIDCR (numbers DE13664 and DE019730, to H.M.F.). We would also like to thank Dr Jon Potempa for the gingipain antibodies.

\section{REFERENCES}

Abaibou, H., Chen, Z., Olango, G. J., Liu, Y., Edwards, J. \& Fletcher, H. M. (2001), vimA gene downstream of recA is involved in virulence modulation in Porphyromonas gingivalis W83. Infect Immun 69, 325335.

Amano, A. (2003). Molecular interaction of Porphyromonas gingivalis with host cells: implication for the microbial pathogenesis of periodontal disease. J Periodontol 74, 90-96.

Amano, A., Sharma, A., Sojar, H. T., Kuramitsu, H. K. \& Genco, R. J. (1994). Effects of temperature stress on expression of fimbriae and superoxide dismutase by Porphyromonas gingivalis. Infect Immun 62, 4682-4685.

Amano, A., Premaraj, T., Kuboniwa, M., Nakagawa, I., Shizukuishi, S., Morisaki, I. \& Hamada, S. (2001). Altered antigenicity in periodontitis patients and decreased adhesion of Porphyromonas gingivalis by environmental temperature stress. Oral Microbiol Immunol 16, 124128.

Aravind, L. \& Koonin, E. V. (1999). Gleaning non-trivial structural, functional and evolutionary information about proteins by iterative database searches. J Mol Biol 287, 1023-1040.

Baev, D., England, R. \& Kuramitsu, H. K. (1999). Stress-induced membrane association of the Streptococcus mutans GTP-binding protein, an essential G protein, and investigation of its physiological role by utilizing an antisense RNA strategy. Infect Immun 67, 4510-4516.

Baev, D., Ohk, S. H. \& Kuramitsu, H. K. (2000). Protein interactions of SGP, an essential Streptococcus mutans GTPase, revealed by biochemical and yeast two-hybrid system analyses. FEMS Microbiol Lett 184, 149-153.

Bronner, S., Monteil, H. \& Prevost, G. (2004). Regulation of virulence determinants in Staphylococcus aureus: complexity and applications. FEMS Microbiol Rev 28, 183-200.

Burland, T. G. (2000). DNASTAR's Lasergene sequence analysis software. Methods Mol Biol 132, 71-91.

Chapple, I. L. (1997). Reactive oxygen species and antioxidants in inflammatory diseases. J Clin Periodontol 24, 287-296.

Curtis, M. A., Slaney, J. M. \& Duse-Opoku, J. (2005). Critical pathways in microbial virulence. J Clin Periodontol 32 (Suppl. 6), 2838 .
Dorman, C. J. (2009). Global regulators and environmental adaptation in Gram-negative pathogens. Clin Microbiol Infect $\mathbf{1 5}$ (Suppl. 1), 47-50.

Fedi, P. F., Jr \& Killoy, W. J. (1992). Temperature differences at periodontal sites in health and disease. J Periodontol 63, 24-27.

Fitzpatrick, R. E., Wijeyewickrema, L. C. \& Pike, R. N. (2009). The gingipains: scissors and glue of the periodontal pathogen, Porphyromonas gingivalis. Future Microbiol 4, 471-487.

Fletcher, H. M., Schenkein, H. A., Morgan, R. M., Bailey, K. A., Berry, C. R. \& Macrina, F. L. (1995). Virulence of a mutant of Porphyromonas gingivalis W83 defective in the prtH gene. Infect Immun 63, 15211528.

Forng, R. Y., Champagne, C., Simpson, W. \& Genco, C. A. (2000). Environmental cues and gene expression in Porphyromonas gingivalis and Actinobacillus actinomycetemcomitans. Oral Dis 6, 351-365.

Geer, L. Y., Domrachev, M., Lipman, D. J. \& Bryant, S. H. (2002). CDART: protein homology by domain architecture. Genome Res 12, 1619-1623.

Hasegawa, Y., Nishiyama, S., Nishikawa, K., Kadowaki, T., Yamamoto, K., Noguchi, T. \& Yoshimura, F. (2003). A novel type of two-component regulatory system affecting gingipains in Porphyromonas gingivalis. Microbiol Immunol 47, 849-858.

Ishiguro, I., Saiki, K. \& Konishi, K. (2009). PG27 is a novel membrane protein essential for a Porphyromonas gingivalis protease secretion system. FEMS Microbiol Lett 292, 261-267.

Jiang, M., Sullivan, S. M., Wout, P. K. \& Maddock, J. R. (2007). Gprotein control of the ribosome-associated stress response protein SpoT. J Bacteriol 189, 6140-6147.

Johnson, N. A., McKenzie, R., McLean, L., Sowers, L. C. \& Fletcher, H. M. (2004). 8-Oxo-7,8-dihydroguanine is removed by a nucleotide excision repair-like mechanism in Porphyromonas gingivalis W83. J Bacteriol 186, 7697-7703.

Katsuragi, H., Ohtake, M., Kurasawa, I. \& Saito, K. (2003). Intracellular production and extracellular release of oxygen radicals by PMNs and oxidative stress on PMNs during phagocytosis of periodontopathic bacteria. Odontology 91, 13-18.

Kedzierska, S., Staniszewska, M., Wegrzyn, A. \& Taylor, A. (1999). The role of DnaK/DnaJ and GroEL/GroES systems in the removal of endogenous proteins aggregated by heat-shock from Escherichia coli cells. FEBS Lett 446, 331-337.

Kesavalu, L., Holt, S. C. \& Ebersole, J. L. (2003). In vitro environmental regulation of Porphyromonas gingivalis growth and virulence. Oral Microbiol Immunol 18, 226-233.

Lamont, R. J. \& Jenkinson, H. F. (1998). Life below the gum line: pathogenic mechanisms of Porphyromonas gingivalis. Microbiol Mol Biol Rev 62, 1244-1263.

Larkin, M. A., Blackshields, G., Brown, N. P., Chenna, R., McGettigan, P. A., McWilliam, H., Valentin, F., Wallace, I. M., Wilm, A. \& other authors (2007). CLUSTAL W and CLUSTAL_X version 2.0. Bioinformatics 23, 2947-2948.

Marmur, J. (1961). A procedure for the isolation of deoxyribonucleic acid from microorganisms. J Mol Biol 3, 208-218.

Mauriello, E. M., Mouhamar, F., Nan, B., Ducret, A., Dai, D., Zusman, D. R. \& Mignot, T. (2010). Bacterial motility complexes require the actin-like protein, MreB and the Ras homologue, MglA. EMBO J 29, 315-326.

Mikolajczyk, J., Boatright, K. M., Stennicke, H. R., Nazif, T., Potempa, J., Bogyo, M. \& Salvesen, G. S. (2003). Sequential autolytic processing activates the zymogen of Arg-gingipain. J Biol Chem 278, 10458-10464.

Murakami, Y., Masuda, T., Imai, M., Iwami, J., Nakamura, H., Noguchi, T. \& Yoshimura, F. (2004). Analysis of major virulence 
factors in Porphyromonas gingivalis under various culture temperatures using specific antibodies. Microbiol Immunol 48, 561-569.

Nakayama, K. (2003). Molecular genetics of Porphyromonas gingivalis: gingipains and other virulence factors. Curr Protein Pept Sci 4, 389 395.

Olango, G. J., Roy, F., Sheets, S. M., Young, M. K. \& Fletcher, H. M. (2003). Gingipain RgpB is excreted as a proenzyme in the vimAdefective mutant Porphyromonas gingivalis FLL92. Infect Immun 71, 3740-3747.

Olarewaju, O., Ortiz, P. A., Chowdhury, W. Q., Chatterjee, I. \& Kinzy, T. G. (2004). The translation elongation factor eEF1B plays a role in the oxidative stress response pathway. RNA Biol 1, 89-94.

Percival, R. S., Marsh, P. D., Devine, D. A., Rangarajan, M., DuseOpoku, J., Shepherd, P. \& Curtis, M. A. (1999). Effect of temperature on growth, hemagglutination, and protease activity of Porphyromonas gingivalis. Infect Immun 67, 1917-1921.

Potempa, J., Mikolajczyk-Pawlinska, J., Brassell, D., Nelson, D., Thøgersen, I. B., Enghild, J. J. \& Travis, J. (1998). Comparative properties of two cysteine proteinases (gingipains R), the products of two related but individual genes of Porphyromonas gingivalis. J Biol Chem 273, 21648-21657.

Potempa, J., Sroka, A., Imamura, T. \& Travis, J. (2003). Gingipains, the major cysteine proteinases and virulence factors of Porphyromonas gingivalis: structure, function and assembly of multidomain protein complexes. Curr Protein Pept Sci 4, 397-407.

Roy, F., Vanterpool, E. \& Fletcher, H. M. (2006). HtrA in Porphyromonas gingivalis can regulate growth and gingipain activity under stressful environmental conditions. Microbiology 152, 33913398.

Saiki, K. \& Konishi, K. (2010). The role of Sov protein in the secretion of gingipain protease virulence factors of Porphyromonas gingivalis. FEMS Microbiol Lett 302, 166-174.
Sambrook, J. \& Russell, D. W. (2001). Molecular Cloning: a Laboratory Manual, 3rd edn. Cold Spring Harbor, NY: Cold Spring Harbor Laboratory.

Sayed, A., Matsuyama, S. \& Inouye, M. (1999). Era, an essential Escherichia coli small G-protein, binds to the 30 S ribosomal subunit. Biochem Biophys Res Commun 264, 51-54.

Scott, J. M. \& Haldenwang, W. G. (1999). Obg, an essential GTP binding protein of Bacillus subtilis, is necessary for stress activation of transcription factor sigma(B). J Bacteriol 181, 4653-4660.

Sculley, D. V. \& Langley-Evans, S. C. (2002). Salivary antioxidants and periodontal disease status. Proc Nutr Soc 61, 137-143.

Seymour, G. J., Ford, P. J., Cullinan, M. P., Leishman, S. \& Yamazaki, K. (2007). Relationship between periodontal infections and systemic disease. Clin Microbiol Infect 13 (Suppl. 4), 3-10.

Sheets, S. M., Potempa, J., Travis, J., Fletcher, H. M. \& Casiano, C. A. (2006). Gingipains from Porphyromonas gingivalis W83 synergistically disrupt endothelial cell adhesion and can induce caspase-independent apoptosis. Infect Immun 74, 5667-5678.

Vanterpool, E., Roy, F. \& Fletcher, H. M. (2004). The vimE gene downstream of $v i m A$ is independently expressed and is involved in modulating proteolytic activity in Porphyromonas gingivalis W83. Infect Immun 72, 5555-5564.

Vanterpool, E., Roy, F. \& Fletcher, H. M. (2005). Inactivation of vimF, a putative glycosyltransferase gene downstream of vimE, alters glycosylation and activation of the gingipains in Porphyromonas gingivalis W83. Infect Immun 73, 3971-3982.

Vanterpool, E., Roy, F., Zhan, W., Sheets, S. M., Sangberg, L. \& Fletcher, H. M. (2006). VimA is part of the maturation pathway for the major gingipains of Porphyromonas gingivalis W83. Microbiology 152, 3383-3389.

Edited by: R. J. Lamont 